\title{
The Nigrostriatal Dopaminergic System as a Preferential Target of Repeated Exposures to Combined Paraquat and Maneb: Implications for Parkinson's Disease
}

\author{
Mona Thiruchelvam, ${ }^{1}$ Eric K. Richfield, ${ }^{2,6}$ Raymond B. Baggs, ${ }^{5,6}$ Arnold W. Tank, ${ }^{3}$ and \\ Deborah A. Cory-Slechta ${ }^{4,6}$ \\ 1/nterdepartmental Program in Neuroscience, Departments of ${ }^{2}$ Pathology and Laboratory Medicine, ${ }^{3}$ Pharmacology and \\ Physiology, and ${ }^{4}$ Environmental Medicine, the ${ }^{5}$ Division of Laboratory Animal Medicine, and the 6 National Institute of \\ Environmental Health Sciences Center, University of Rochester School of Medicine and Dentistry, Rochester, New York 14642
}

\begin{abstract}
Experimental evidence supporting 1,1'-dimethyl-4,4'bipyridinium [paraquat (PQ)] as a risk factor for Parkinson's disease (PD) is equivocal. Other agricultural chemicals, including dithiocarbamate fungicides such as manganese ethylenebisdithiocarbamate [maneb (MB)], are widely used in the same geographical regions as paraquat and also impact dopamine systems, suggesting that mixtures may be more relevant etiological models. This study therefore proposed that combined $P Q$ and MB exposures would produce greater effects on dopamine (DA) systems than would either compound administered alone. Male C57BL/6 mice were treated twice a week for 6 weeks with intraperitoneal saline, $10 \mathrm{mg} / \mathrm{kg}$ paraquat, $30 \mathrm{mg} / \mathrm{kg}$ maneb, or their combination $(P Q+M B)$. $M B$, but not $P Q$, reduced motor activity immediately after treatment, and this effect was potentiated by combined $\mathrm{PQ}+\mathrm{MB}$ treatment. As treatments progressed, only the combined $P Q+M B$ group evidenced a failure
\end{abstract}

of motor activity levels to recover within $24 \mathrm{hr}$. Striatal DA and dihydroxyphenylacetic acid increased 1-3 d and decreased $7 \mathrm{~d}$ after injections. Only $P Q+M B$ reduced tyrosine hydroxylase (TH) and DA transporter immunoreactivity and did so in dorsal striatum but not nucleus accumbens. Correspondingly, striatal $\mathrm{TH}$ protein levels were decreased only by combined $\mathrm{PQ}+\mathrm{MB}$ $5 \mathrm{~d}$ after injection. Reactive gliosis occurred only in response to combined $\mathrm{PQ}+\mathrm{MB}$ in dorsal-medial but not ventral striatum. TH immunoreactivity and cell counts were reduced only by $P Q+M B$ and in the substantia nigra but not ventral tegmental area. These synergistic effects of combined $P Q+M B$, preferentially expressed in the nigrostriatal DA system, suggest that such mixtures could play a role in the etiology of PD.

Key words: dopamine; striatum; nucleus accumbens; substantia nigra; tyrosine hydroxylase; dopamine transporter; locomotor activity; gliosis
Parkinson's disease (PD), a profound movement disorder resulting from nigrostriatal dopaminergic (DA) system degeneration, has been linked to living in a rural environment, farming, drinking well water, and occupational exposure to agricultural chemicals, suggesting an environmental exposure basis for the disease (Rajput et al., 1987; Semchuk et al., 1992; Liou et al., 1997; Gorell et al., 1998; Tanner et al., 1999). Correspondingly, a recent comprehensive study of over 19,000 white male twins showed that genetic heritability is not the basis of sporadic PD with onset over age 50 (Tanner et al., 1999). The identification of MPTP, a synthetic heroin that destroys substantia nigra DA neurons, has given additional credence to an environmental factor hypothesis (Langston et al., 1984; Langston and Irwin, 1986; Tanner and Ben-Shlomo, 1999). MPTP, however, is not found in the environment.

The herbicide 1,1'-dimethyl-4,4'-bipyridinium [paraquat (PQ)] has emerged as a putative risk factor on the basis of its structural homology to $\mathrm{MPP}^{+}$, the active metabolite of MPTP. Occupational PQ exposures have been associated with parkinsonism (Hertzman et al., 1990; Liou et al., 1997). Although substantially impeded, PQ does cross the blood-brain barrier, with higher levels evident $24 \mathrm{hr}$ as compared with 30 min after administration (Widdowson et al., 1996a).

\footnotetext{
Received Aug. 22, 2000; revised Sept. 25, 2000; accepted Sept. 27, 2000.

This work was supported in part by National Institute of Environmental Health Sciences Grants ES01247, ES05017, and ES05903 to D.A.C.-S., Department of Defense Grant DMD17-98-1-8628 to E.K.R., and National Institute on Drug Abuse Grant DA05014 and Tobacco Research Council Grant STRC0481 to A.W.T. Special thanks go to Dr. Kenneth Olden. We also thank Brian Rahm and Carol Sterling for technical assistance.

Correspondence should be addressed to Dr. Deborah A. Cory-Slechta, Department of Environmental Medicine, Box Environmental Health Sciences Center, University of Rochester School of Medicine and Dentistry, 601 Elmwood Avenue, Rochester, NY 14642. E-mail: deborah_slechta@urmc.rochester.edu.

Copyright (C) 2000 Society for Neuroscience $0270-6474 / 00 / 209207-08 \$ 15.00 / 0$
}

$\mathrm{PQ}$, however, is a member of only one class of agricultural chemicals known to impact DA systems adversely and to be used in overlapping geographical areas. Diethyldithiocarbamate fungicides potentiate MPTP neurotoxicity (Miller et al., 1991; Walters et al., 1999). Manganese ethylenebisdithiocarbamate [maneb (MB)], for example, decreases locomotor activity (Morato et al., 1989) and potentiates MPTP effects on locomotor activity and catalepsy (Takahashi et al., 1989). Interestingly, at least two incidences of parkinsonism in humans have been related to MB exposure (Ferraz et al., 1988; Meco et al., 1994). The heaviest use of both PQ and diethyldithiocarbamates like MB occurs along the Pacific Coast and in the Northeast, the Plains, the mid-Atlantic, the Southeast states, and Texas (United States Geographic Service, 1998). PQ and diethyldithiocarbamates are also used on many of the same crops [e.g., tomatoes (Wilhoit et al., 1999)]. The geographical overlap in use patterns and the fact that multiple pesticide residues can be found in foods suggest that human exposures are to agrichemical mixtures, raising the possibility of multiple-hit models of PD. In such models, exposure to an individual chemical may be insufficient to induce overt effects, whereas multiple concurrent exposures, by provoking changes at multiple target sites of the nigrostriatal DA system, could preclude the operation of homeostatic defense mechanisms leading to neuronal cell death.

On the basis of this premise, we examined the hypothesis that combined $\mathrm{PQ}$ and $\mathrm{MB}(\mathrm{PQ}+\mathrm{MB})$ exposures would produce additive effects on the nigrostriatal DA system. A previous experiment from our laboratory provided initial but limited support for this assertion (Thiruchelvam et al., 2000). Combined PQ + MB administered to mice once a week for 4 weeks potentiated reductions in locomotor activity and increased striatal DA, its metabolites, and DA turnover. It also reduced tyrosine hydroxylase (TH) density relative to either compound administered alone and did so in the dorsal striatum but not the nucleus accumbens, suggesting a 
targeting of the nigrostriatal DA system. The current study sought to validate this model and to determine the selectivity and sensitivity of combined $\mathrm{PQ}+\mathrm{MB}$ for the nigrostriatal system, the impact of exposure duration, and the involvement of substantia nigra DA cell bodies. Collectively the findings are consistent with the conclusions that combined $\mathrm{PQ}+\mathrm{MB}$ exposure is preferentially neurotoxic to the nigrostriatal DA system, that this effect occurs via enhanced DA activity, and that such combined exposures may serve as environmental risk factors for PD.

\section{MATERIALS AND METHODS}

\section{Animals}

Male C57BL/6 mice 6 weeks of age purchased from Taconic (Germantown, NY) were housed in a room maintained under constant temperature $\left(72-74^{\circ} \mathrm{F}\right)$ and humidity conditions with a $12 / 12 \mathrm{hr}$ light/dark cycle. Fivemonth-old male C57BL/6 mice used in the studies to determine the extent and nature of neurotoxicity associated with combined PO and MB were purchased from the National Institute on Aging aging colony (Harlan Sprague Dawley, Indianapolis, IN). Those used for behavioral studies were housed one per cage; all other mice were housed five per cage. Food and water were available ad libitum. Mice were habituated to the vivarium for at least 1 week before commencement of experiments. Body weights were obtained daily over the course of the experiment. Animals were cared for and treated in accord with National Institutes of Health and the University of Rochester Animal Care and Use Committee guidelines.

\section{Chemicals}

Solvents for HPLC with electrochemical detection (HPLC-EC) were purchased from Sigma (St. Louis, MO). All other chemicals, if not specified, were at least analytical grade and were purchased from Sigma.

\section{Drug administration}

Mice were injected intraperitoneally with either saline (vehicle), $10 \mathrm{mg} / \mathrm{kg}$ paraquat dichloride hydrate (Sigma), $30 \mathrm{mg} / \mathrm{kg}$ maneb (gift from DuPont Agricultural Products), or PQ + MB. A range of $L_{50}$ values for PQ has been reported for mice, extending from 30 to $60-70 \mathrm{mg} / \mathrm{kg}$ and suggesting that strain, gender, and solution factors are important (Bus et al., 1976; Drew and Gram, 1979; Yamamoto, 1993). Corresponding values for MB in mice are not reported, although $\mathrm{LD}_{50}$ values for oral administration in rats range from 4.5 to $6.7 \mathrm{gm} / \mathrm{kg}$ (Berg, 1977). Both PQ and MB were dissolved in saline. Mice were injected twice a week for 6 weeks for a total of 12 injections. For combined injections, two separate injections were administered. Animals were killed at varying time points, ranging from $1 \mathrm{hr}$ to $7 \mathrm{~d}$ after the last treatment.

\section{Locomotor activity}

Automated locomotor activity chambers equipped with infrared photobeams (Opto-Varimex Minor; Columbus Instruments International Corporation, Columbus, $\mathrm{OH}$ ) were used to quantify locomotor activity. Photobeam breaks were recorded each minute for $45 \mathrm{~min}$ for horizontal, vertical, and ambulatory movements. Mice were initially habituated to the locomotor activity chambers in three 45 min sessions occurring on consecutive days, with all mice receiving intraperitoneal vehicle injections before the session. After the third habituation session, treatments began, and effects on motor activity were assessed immediately and $24 \mathrm{hr}$ after each injection (sessions 1 and 2, respectively) in 45 min test sessions with activity counts totaled in 3 min blocks across the session.

\section{MPTP challenge}

In a separate group of animals, 1 week after the 12 th treatment, animals from each treatment group (i.e., saline, $\mathrm{PQ}, \mathrm{MB}$, or $\mathrm{PQ}+\mathrm{MB}$ ) were treated with either saline or $15 \mathrm{mg} / \mathrm{kg} \mathrm{MPTP}(n=5)$. Locomotor activity was assessed either immediately (see Fig. 3) or $24 \mathrm{hr}$ after treatment (data not shown).

\section{Permanent, progressive, or reversible effects of $P Q+$ $M B$ neurotoxicity}

To address the issue of reversibility of the adverse effects of combined PQ and $\mathrm{MB}, 5$-month-old mice were treated twice a week for 3 weeks with either saline, $10 \mathrm{mg} / \mathrm{kg} \mathrm{PQ}, 30 \mathrm{mg} / \mathrm{kg} \mathrm{MB}$, or PQ + MB ( $n=6$ per group). Three months after the last injection, animals were killed, brains were removed, and tyrosine hydroxylase protein levels were determined using Western blot analysis (as described below).

\section{Dopamine and metabolite analyses by HPLC}

Neurotransmitter concentrations were measured $1 \mathrm{hr}, 3 \mathrm{~d}$, and $7 \mathrm{~d}$ after the 12th injection of either saline, $\mathrm{PQ}, \mathrm{MB}$, or $\mathrm{PQ}+\mathrm{MB}$. After rapid decapitation, striatal sections were dissected and placed in $0.1 \mathrm{~N}$ perchloric acid. The tissues were sonicated and centrif uged for $8 \mathrm{~min}$ at $1000 \times \mathrm{g}$. The supernatants were stored at $-80^{\circ} \mathrm{C}$ until analyzed for the concentrations of DA, dihydroxyphenylacetic acid (DOPAC), and homovanillic acid by
HPLC-EC. The pellets were digested in $1 \mathrm{ml}$ of $0.5 \mathrm{~N} \mathrm{NaOH}$ for measurements of protein concentration using Bio-Rad (Hercules, CA) assay reagents. For HPLC-EC analysis, samples were loaded onto a Waters 717 plus autosampler (Waters Associates, Milford, MA), and the mobile phase was delivered at a constant rate of $1 \mathrm{ml} / \mathrm{min}$ by a Waters model 510 pump through a C18, $5 \mathrm{~mm}, 250 \times 4.6 \mathrm{~mm}$ analytical column (Alltech, Deerfield, IL) placed in a column heater $\left(35^{\circ} \mathrm{C}\right)$. The $\mathrm{LC}$ amperometric potential was set to $0.75 \mathrm{~V}$ with reference to an $\mathrm{Ag}-\mathrm{AgCl}$ reference electrode, and the sensitivity of the detector was maintained at $2 \mathrm{nA}$. The mobile phase consisted of $0.1 \mathrm{~m}$ disodium phosphate, $0.1 \mathrm{~m}$ citrate, $0.15 \mathrm{~mm}$ EDTA, 1.4 $\mathrm{mm}$ octyl sodium sulfate, and $12 \%$ methanol. The signal from the detector was recorded, and the data were analyzed by the use of a Waters Millennium 2010 Chromatography Manager. The concentrations of the neurotransmitters were expressed as nanograms per milligram of protein. DA turnover was expressed as the ratio DOPAC/DA.

\section{Immunohistochemistry for tyrosine hydroxylase and the dopamine transporter}

Tissue preparation. After intracardial perfusion with heparinized saline followed by $4 \%$ paraformaldehyde (PFA), brains were harvested and post-fixed in $4 \%$ PFA for $2 \mathrm{hr}$ and consequently cryoprotected in buffered sucroses to $30 \%$ over $2 \mathrm{~d}$. Brains were cut into $40 \mu \mathrm{m}$ sections and collected in cryoprotectant. Sections were stored at $-20^{\circ} \mathrm{C}$ until used for immunohistochemistry (IHC).

Immunohistochemistry. Sections were washed with $0.1 \mathrm{M}$ phosphate buffer (PB), blocked for nonspecific binding, and incubated with a $1^{\circ}$ antibody (Ab) either to TH (Chemicon, Temecula, CA) or to the DA transporter (Chemicon) overnight at a dilution of 1:4000 in $0.1 \mathrm{M} \mathrm{PB}$ containing $0.3 \%$ Triton X-100 and $10 \%$ goat serum. Sections were subsequently washed and incubated with avidin-biotin solution using the Vectastain Elite kit (Vector Laboratories, Burlingame, CA) for $1 \mathrm{hr}$ at room temperature. Sections were quenched for endogenous peroxidase by the use of hydrogen peroxide for $10 \mathrm{~min}$. Sections were then developed in 3-3'-diaminobenzidine tetrachloride for 2-3 min. After several rinses, sections were mounted out of PB, dehydrated, and coverslipped.

\section{Immunohistochemistry and astrocyte counts for glial fibrillary acidic protein}

Tissue preparation was similar to that described for TH and the dopamine transporter (DAT). Sections were washed with $0.1 \mathrm{M}$ PB, blocked, and incubated with anti-glial fibrillary acidic protein (anti-GFAP; Chemicon) at a dilution of $1: 500$ overnight at $4^{\circ} \mathrm{C}$. Sections were then washed and incubated with an anti-rabbit Alexa $594 \mathrm{Ab}$ (Molecular Probes, Eugene OR) at a dilution of 1:500 for $2 \mathrm{hr}$. Sections were then washed and mounted with mowiol (Polysciences, Warrington, PA). Fluorescent images were viewed using a TRITC filter, and reactive astrocytes were counted without the experimenter having knowledge of the treatment. The total number of clusters and total number of astrocytes were quantitated in the dorsalmedial striatum ( $n=6$ per treatment group). Clusters were defined as two or more reactive astrocytes adjacent to each other. Only reactive astrocytes expressing abundant GFAP were counted. Astrocytes that were close to white matter or were entangled with vasculature were omitted. The average numbers of astrocytes and clusters per section from each animal were used for statistical analysis.

\section{Densitometric measurements of tyrosine hydroxylase and dopamine transporter immunoreactivity}

Six serial striatal sections and four nucleus accumbens sections were used for densitometric measurements, with each treatment group represented by an $n=6$. Densitometric measurements were performed using NIH Image software and with the experimenter blinded to the treatment groups. Images were acquired at the same magnification to capture the entire striatal area in a single field and converted to gray scale before the staining intensity was analyzed. Two different areas from each half of a section, encompassing $30 \%$ of the total area, were analyzed to ensure adequate representation of the entire area of interest. The density of the two regions was averaged to give a total mean density. Background was established by determining the density of an area that did not stain with anti-TH antibody.

\section{Tyrosine hydroxylase protein levels by the use of Western blot analysis}

After rapid decapitation, the striatum was removed and stored at $-80^{\circ} \mathrm{C}$ until analysis. Protein concentrations were determined using the Bio-Rad assay reagents. Each treatment group had an $n=8$. For each sample, three different concentrations of protein $(5,10$, and $20 \mu \mathrm{g}$ ) were loaded onto separate lanes. The samples were subjected to electrophoresis, transferred to nitrocellulose, and immunoblotted with rabbit antiserum specific for TH. Detection was performed with the ECL kit using autoradiography. The autoradiograms were scanned, and the autoradiographic bands were quantitated using NIH Image software to calculate the density. Only density values that were within the linear range of the autoradiographic film were used (Osterhout et al., 1997). The density for each TH protein band was normalized to the amount of protein loaded onto the gel for that 

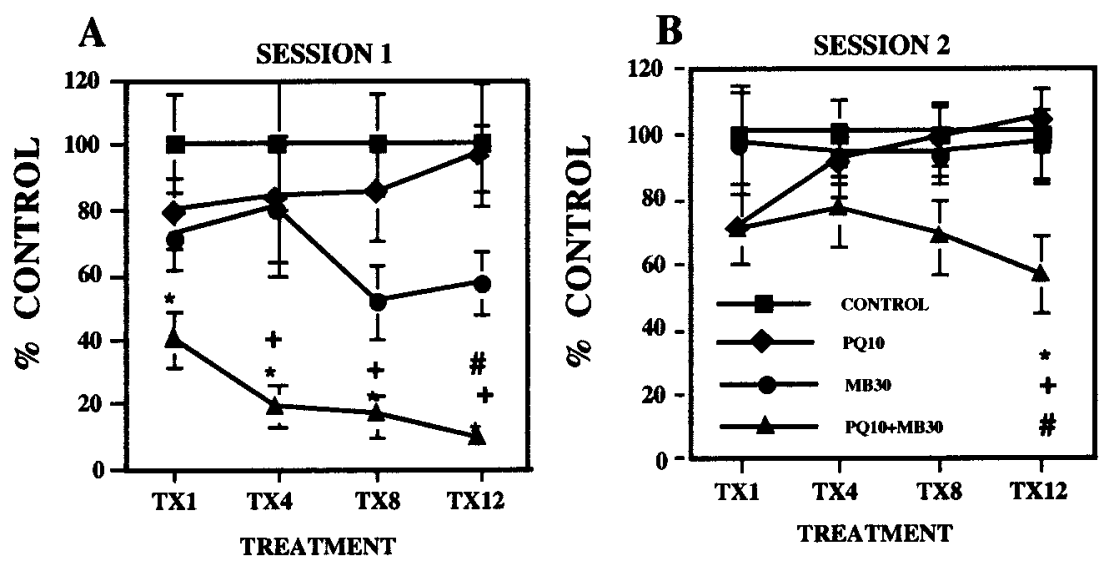

Figure 1. A, Total ambulatory locomotor activity. The group mean $( \pm \mathrm{SE} ; n=10$; plotted as percent of control group values) was measured immediately after the 1 st, 4 th, 8 th, and 12 th injections ( $T X 1, T X 4, T X 8$, and $T X 12$, respectively) of either saline (CONTROL), $10 \mathrm{mg} / \mathrm{kg}$ paraquat $(P O 10), 30 \mathrm{mg} / \mathrm{kg}$ maneb $(M B 30)$, or their combination $(P Q 10+M B 30)$. Bonferroni-Dunn post hoc tests for each treatment day indicate differences: *, from the control group; +, from $\mathrm{PQ}$ alone; \#, from $\mathrm{MB}$ alone. $B$, Corresponding effects measured $24 \mathrm{hr}$ after treatment. particular sample and then divided by the density of the known amount of $\mathrm{TH}$ protein loaded onto that gel. TH protein values were expressed as micrograms of TH protein per milligram of protein loaded.

\section{Tyrosine hydroxylase staining intensity in the substantia nigra and ventral tegmental area}

Sections were stained as described under TH immunohistochemistry. Quantitative estimates of TH immunoreactivity were made in the bilateral substantia nigra pars compacta $(\mathrm{SNc})$ and the ventral tegmental area (VTA). Intensity measurements were done with the experimenter blinded to the treatment and in random order to avoid any bias. A total of five representative sections per animal were used to determine TH density within the SNc, and two sections per animal were used for the VTA. Each treatment group was represented by an $n=6$. The analysis, using the NIH Image software program, was done after encircling the SNc or VTA in each sampled section at a magnification of $40 \times$, permitting representative visualization of each region. These parameters were constant across sampled sections. The average of the density for each section was added and divided by the total number of sections analyzed to give the mean $\mathrm{TH}$ density for each animal.

\section{Cell counts in the substantia nigra and ventral tegmental area}

The sampled sections from all of the animals ( $n=6$ per treatment group) were stained simultaneously by the use of the above described method for TH IHC. After being mounted on gelatin-coated slides, the sections were counterstained with cresyl violet to allow the visualization of TH-positive neuronal nucleoli. Three representative sections containing the SNc and the VTA were chosen. Quantitative counts of the total number of THimmunoreactive cell bodies counterstained with cresyl violet $(\mathrm{TH} /$ cresyl violet-positive neurons) were made in the bilateral SNc and VTA. Counts were performed manually and with the experimenter blinded to the treatment received. The average number of neurons per section was added to provide a measure of the total number of $\mathrm{TH} /$ cresyl violet-positive neurons for each animal and then divided by the number of counted sections to give a mean of the number of labeled neurons per section.

\section{Lung histopathology}

Because PQ is known to target lung (Bus et al., 1976), representative sections of lung $(n=6)$ were prepared by formalin fixation, paraffin embedding, sectioning at $4 \mu \mathrm{m}$, and staining with hematoxylin and eosin. Sections were examined without knowledge of the treatment group for evidence of alterations in alveoli, respiratory ducts, bronchioles, and bronchi.

\section{Statistical analysis}

Overall effects of treatment on horizontal locomotor activity were first analyzed with repeated measure ANOVAs (RMANOVAs) using treatment as a between-groups factor and injections as a within-group factor. This was followed by individual one-way ANOVAs using treatment as a between-groups factor for each injection (see Fig. 1) and subsequent post hoc Bonferroni-Dunn tests to compare treatment groups. BonferroniDunn tests control for numbers of comparisons and thus provide a conservative estimate of significance. To assess treatment-related changes within an activity session (see Fig. 2), RMANOVAs with treatment as a between-groups factor and time block as a within-group factor were used; significant main effects of treatment or interactions were followed by one-factor ANOVAs at each time point. Changes in DA, DOPAC, and turnover (see Fig. 4) were first evaluated using treatment and time point (1 $\mathrm{hr}, 3 \mathrm{~d}$, and $7 \mathrm{~d}$ ) as between-groups factors for ANOVA. This was followed by separate ANOVAs for each time point for each measure. Effects of all other end points were analyzed using one-factor ANOVA with treatment as the between-groups factor, followed by Bonferroni-Dunn tests in the event of significant main effects of treatment.

\section{RESULTS}

\section{Body weight and lung pathology}

No treatment-related changes in body weights were observed at any point in the experiments, either (1) when body weights before each motor activity session were compared across the entire experiment or (2) when body weights from the final habituation session before treatment were compared with (a) body weights on the 12 injection days, (b) body weights on the day after each injection, or (c) body weights on the last day of the experiment. Lungs were graded for signs of alveolitis, bronchiolitis, bronchitis, lymphoid aggregation, bronchiectasis, and fibrosis and found to be histologically normal.

\section{Locomotor activity}

Total ambulatory activity counts determined immediately after injections (Fig. $1 A$, session 1 ) were reduced only by $\mathrm{PQ}+\mathrm{MB}$ [main effect of treatment, $F_{(3,36)}=4.11(p=0.0132) ; F=4.26$ $(p=0.0113) ; F=8.62(p=0.0002)$; and $F=12.39(p<0.0001)$, for treatments $1,4,8$, and 12 , respectively]. By the 12 th injection, the motor activity levels of the combined PQ $+\mathrm{MB}$ group were significantly lower than were those of all other groups, having decreased to $9 \%$ of control. Although an emerging reduction in activity in response to $\mathrm{MB}$ alone is suggested after treatments 8 and 12 , these effects were not statistically different from control at either time point.

An emergent failure in the recovery of motor activity measured $24 \mathrm{hr}$ after injection was also observed in the combined PQ $+\mathrm{MB}$ group (Fig. $1 B$ ). Although recovery was complete after the 1st, 4 th, and 8th injections, values of the PQ + MB group evidenced only partial recovery by the 12 th injection [main effect of treatment, $\left.F_{(3,36)}=3.52(p=0.0245)\right]$ and were significantly lower than those of the PQ-only group and marginally lower than those of the control and MB-only groups.

Potentiated effects of combined PQ + MB were especially evident across the course of behavioral sessions. As shown after the 4th and 12th injections (Fig. 2A,C), $\mathrm{PQ}+\mathrm{MB}$ significantly reduced motor activity relative to that of the control and PQ-alone groups (not indicated on figure) at virtually every time point and relative to that of the MB-only group during the final half of the session at both time points [interactions of time by treatment, $F_{(42,504)}=2.23(p<$ $0.0001)$; and $F=3.14$ ( $p<0.0001)$, respectively]. Parallel changes were noted in both horizontal and vertical motor activity (data not shown). A suggestion of lower motor activity levels during the first half of the session after the 4th MB-alone injections was enhanced with continuing treatment, producing significant reductions in motor activity relative to that in controls with recovery to control levels during the final half of the session after the 12th injection.

Within $24 \mathrm{hr}$ after treatment 4 (Fig. 2B), all groups exhibited full recovery. By the 12 th injection, however, the combined $\mathrm{PQ}+\mathrm{MB}$ group was no longer exhibiting full recovery of motor activity within $24 \mathrm{hr}$ [Fig. $2 D$, main effect of treatment, $\left.F_{(3,14)}=3.52(p=0.0245)\right]$, with levels now remaining at only $\sim 50 \%$ of those of controls. 
Figure 2. Ambulatory locomotor activity across a behavioral session. The group mean $( \pm \mathrm{SE})$ was measured in 3 min blocks across a 45 min behavioral session immediately after the 4th and 12th treatment $(A, C$, respectively) or $24 \mathrm{hr}$ after treatment $(B, D$, respectively) with vehicle (CONTROL), $10 \mathrm{mg} / \mathrm{kg}$ PQ (PQ10), $30 \mathrm{mg} / \mathrm{kg} \mathrm{MB} \mathrm{(MB30),} \mathrm{or} \mathrm{their}$ combination $(P Q 10+M B 30)$. Significant differences are as follows: *, from the control group; \#, from maneb alone. Significant differences of the combined PQ + MB group from $\mathrm{PQ}$ alone are not shown. Sample sizes are as noted in the Figure 1 legend.
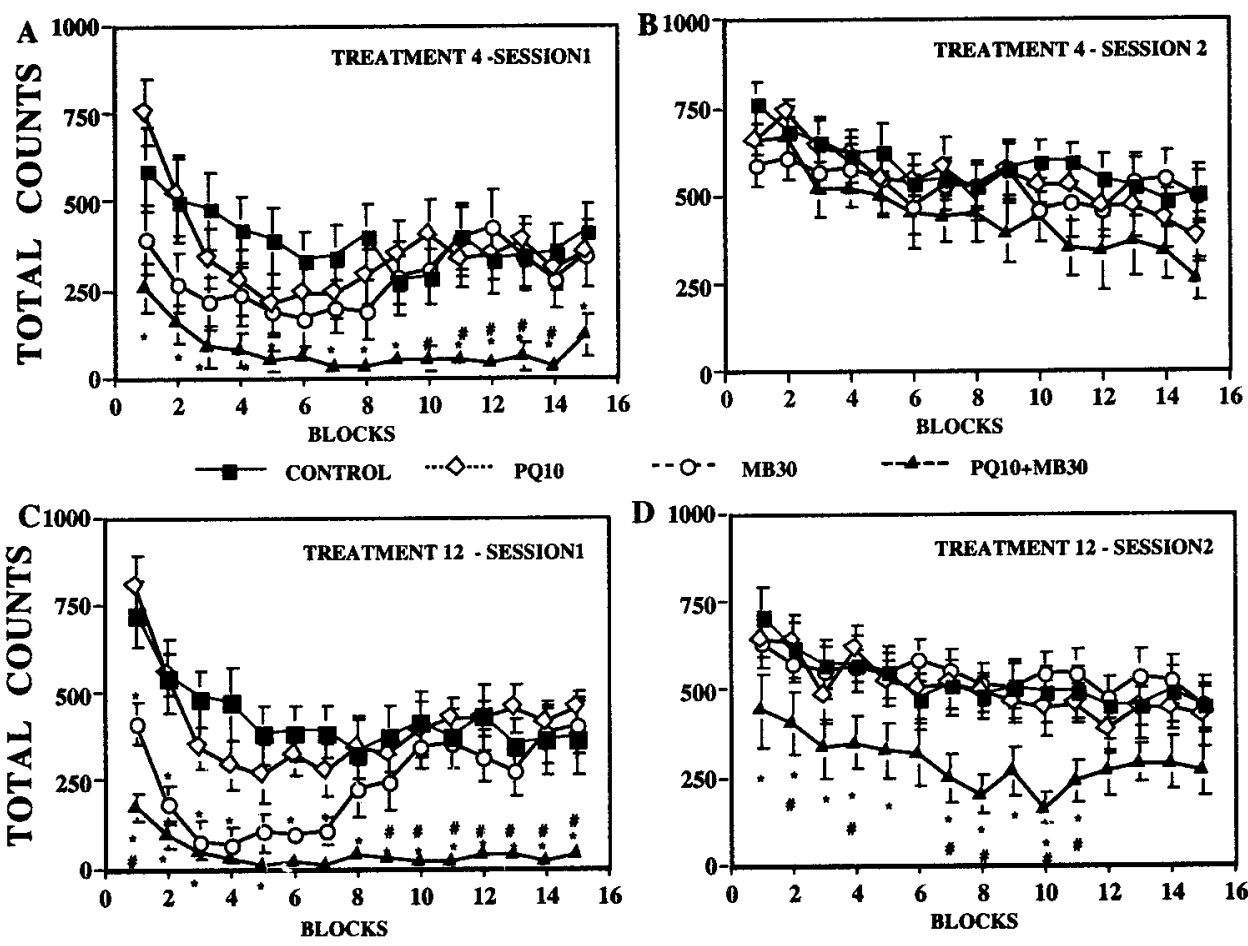

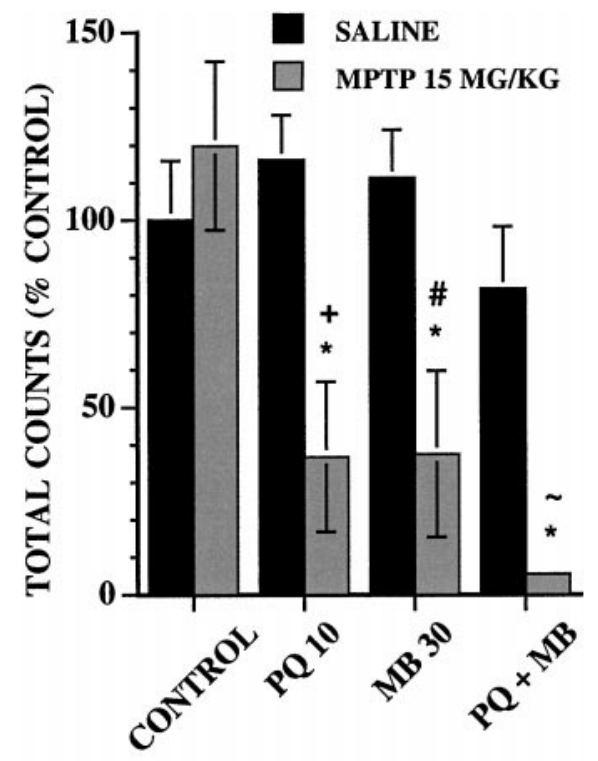

TREATMENT

Figure 3. Effect of an acute dose of MPTP on locomotor activity. The group mean $( \pm \mathrm{SE})$ of total ambulatory locomotor activity counts in $45 \mathrm{~min}$ sessions was measured immediately after intraperitoneal injections of saline $(n=5)$ or $15 \mathrm{mg} / \mathrm{kg}$ MPTP $(n=5)$ after 12 treatments with vehicle (CONTROL), $10 \mathrm{mg} / \mathrm{kg}$ PQ (PQ 10), $30 \mathrm{mg} / \mathrm{kg} \mathrm{MB}$ (MB 30), or their combination $(P Q+M B)$. Data are plotted as the percent of the saline control group values. Bonferroni-Dunn post hoc tests for each treatment day indicate differences: *, from the control saline group; +, from the PQ saline group; \#, from the MB saline group; $\sim$, from the PQ +MB saline group.

\section{MPTP challenge}

Total locomotor activity counts after saline or a challenge dose of $15 \mathrm{mg} / \mathrm{kg}$ MPTP (Fig. 3) were modulated by treatment [main effect of MPTP, $F_{(3,16)}=8.11(p=0.0016)$; treatment by MPTP interaction, $\left.F_{(3,31)}=4.74(p=0.0078)\right]$. This dose of MPTP did not affect motor activity levels of controls. MPTP significantly reduced activity by $63-64 \%$ relative to control in both the PQalone and MB-alone groups. Although the largest decline was found in the PQ + MB group (95\%), it was not significantly greater than that detected in the PQ-alone and MB-alone groups.

\section{Reversibility of $P Q+M B$ neurotoxicity}

Three months after the last treatment of PQ or MB alone, striatal $\mathrm{TH}$ protein levels were comparable with those of saline-treated animals. However, mice treated with $\mathrm{PQ}+\mathrm{MB}$ showed a $39 \%$ decrease in striatal $\mathrm{TH}$ protein levels relative to that in saline controls [main effect of treatment, $F_{(3,20)}=5.555(p=0.0061)$ ]. $\mathrm{TH}$ protein levels of the PQ $+\mathrm{MB}$ group were significantly lower than those in control, PQ-alone, and MB-alone groups in post hoc analysis ( $p=0.004,0.001$, and 0.02 , respectively).

\section{Striatal dopamine, DOPAC, and turnover}

Changes in levels of DA, DOPAC, and DA turnover, measured at $1 \mathrm{hr}, 3 \mathrm{~d}$, and $7 \mathrm{~d}$ after the 12th injection (Fig. 4), differed in relation to time point $\left[F_{(6,70)}=3.20(p=0.0078) ; F=4.66(p=0.0005)\right.$; and $F=3.83(p=0.0023)$, for DA, DOPAC, and DA turnover, respectively]. Subsequent one-factor ANOVAs at each time point for each measure revealed that levels of DA were significantly elevated by $20-30 \% 1 \mathrm{hr}$ after injection in the PQ-alone, MBalone, and $\mathrm{PQ}+\mathrm{MB}$ groups. Values in the $\mathrm{PQ}+\mathrm{MB}$ group, although somewhat higher, differed only marginally from the PQalone and MB-alone groups ( $p=0.09$ and 0.10 , respectively). Corresponding increases in DOPAC at $1 \mathrm{hr}$ were produced by MB alone and by $\mathrm{PQ}+\mathrm{MB}$, with $\mathrm{PQ}+\mathrm{MB}$ group values $(>40 \%$ control) significantly higher than the corresponding control and PQ-alone group values. DA turnover was increased at $1 \mathrm{hr}$ only in the PQ + MB group, at least relative to PQ alone.

Three days after the 12th treatment, residual increases in DA levels differed only marginally from control $(p=0.06)$, because of both the MB-alone $(p=0.014)$ and the PQ + MB groups $(p=$ 0.032 ). DOPAC had recovered to control levels. DA turnover was significantly suppressed at this time point only in the PQ $+\mathrm{MB}$ group [main effect of treatment, $F_{(3,27)}=5.40(p=0.0048)$ ], compared with both the control and PQ-only groups $(p=0.0007$ and 0.005 , respectively).

By day 7, a trend toward reductions of DA, DOPAC, and turnover was suggested and was marginally significant for DOPAC $(p=0.085)$ because of differences between controls and the PQ + MB group (100 vs 73\%; $p=0.014)$. 


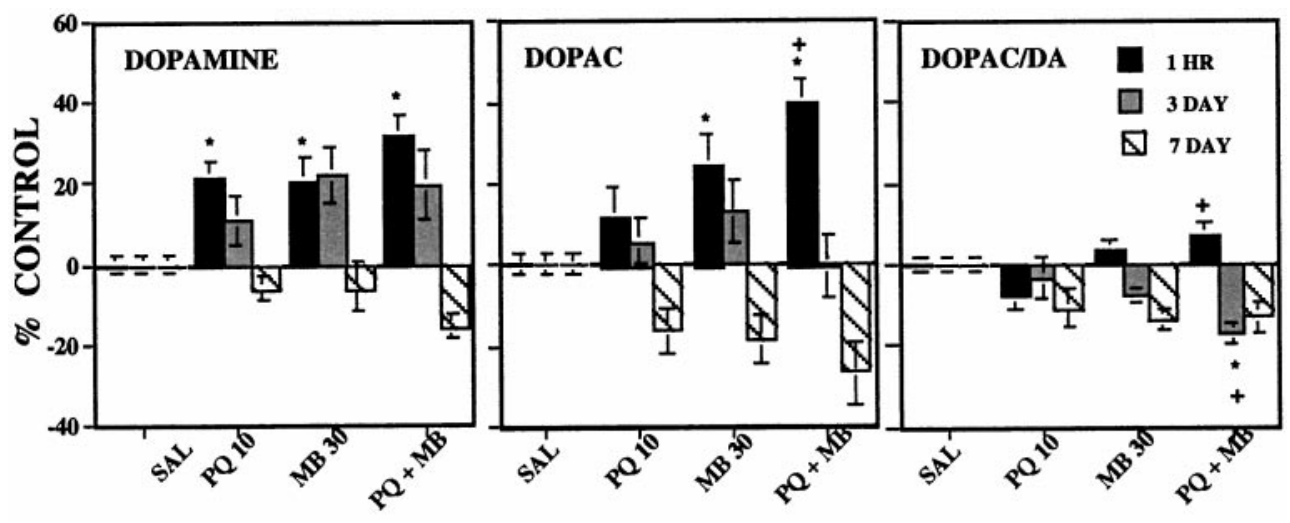

TREATMENT
Figure 4. Striatal levels of DA (left), DOPAC (middle), and DA turnover (DOPAC/DA; right). The changes in group mean ( \pm SE) from control levels of DA, DOPAC, and DA turnover were assessed either $1 \mathrm{hr}(n=10), 3 \mathrm{~d}(n=10)$, or $7 \mathrm{~d}$ $(n=5)$ after the 12th intraperitoneal injection of vehicle $(S A L), 10 \mathrm{mg} / \mathrm{kg}$ paraquat $(P Q 10), 30 \mathrm{mg} / \mathrm{kg}$ maneb $(M B 30)$, or their combination $(P Q+M B)$. Data are plotted as a percent of the control group value for the corresponding time point. Bonferroni-Dunn post hoc analysis indicates significant differences: *, from the control group; +, from PQ alone; \#, from MB alone.
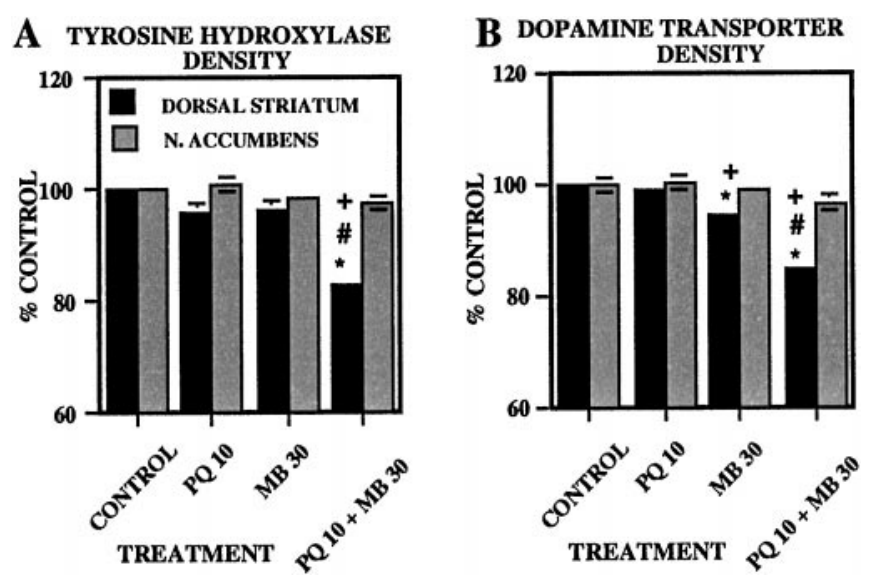

Figure 5. Striatal immunohistochemistry of TH and DAT. Group mean $( \pm \mathrm{SE}$ ) levels of TH $(A)$ and DAT $(B)$ immunoreactivity (expressed as percent of control group values) in dorsal striatum and nucleus $(N$.) accumbens were measured $5 \mathrm{~d}$ after 12 treatments with vehicle (CONTROL; $n=$ $6), 10 \mathrm{mg} / \mathrm{kg}$ paraquat $(P Q 10 ; n=6), 30 \mathrm{mg} / \mathrm{kg}$ maneb $(M B 30 ; n=6)$, or their combination $(P Q 10+M B 30 ; n=6)$. Bonferroni-Dunn post hoc analysis indicates significant differences: *, from the control group; +, from $\mathrm{PQ}$ alone; \#, from MB alone.

\section{Striatal TH and dopamine transporter immunoreactivity}

Levels of TH and DAT immunoreactivity determined $5 \mathrm{~d}$ after the 12th injection (Fig. 5) were reduced $15-17 \%$ by $P Q+$ MB relative to the levels in all other groups in the dorsal striatum $\left[F_{(3,20)}=24.7\right.$ $(p<0.0001)$; and $F=54.1(p<0.0001)$, respectively; BonferroniDunn tests, all $p$ values $<0.0001$, whereas no effects of treatment were detected in the nucleus accumbens $(p=0.18$ and 0.22 , respectively). Striatal DAT immunoreactivity was also reduced $\sim 6 \%$ by $\mathrm{MB}$ alone relative to the control and PQ-only groups ( $p=$ 0.0005 and 0.0032 , respectively).

\section{TH protein levels}

Striatal TH protein levels (Fig. $6 B$ ) were unaffected by treatment 1 $\mathrm{hr}$ after the 12th injection. However, by the $5 \mathrm{~d}$ time point, $\mathrm{PQ}+$ MB treatment had reduced TH by $\sim 35 \%$ [main effect of treatment, $\left.F_{(3,16)}=4.46(p=0.0185)\right]$. These values were significantly lower than were those of both the control and PQ-only groups ( $p=0.005$ and 0.007 , respectively), as evident in the corresponding bands from Western blots (Fig. 6A), particularly at the higher proteinloading levels.

\section{TH immunoreactivity and cell counts in the substantia nigra and ventral tegmental area}

Levels of TH intensity and cell counts $5 \mathrm{~d}$ after the 12th injection (Fig. 7) were reduced only by PQ $+\mathrm{MB}(\sim 32 \%)$ relative to all other groups in the substantia nigra [main effect of treatment, $F_{(3,20)}=32.23(p<0.0001)$; Bonferroni-Dunn tests, all $p$ values $<$
0.0001], whereas there were no effects in the ventral tegmental area $\left[F_{(3,20)}=0.21(p=0.89)\right]$. Cell counts were reduced only by PQ + $\mathrm{MB}$ and only in the substantia nigra [main effect of treatment, $F_{(3,20)}=3.39(p=0.038) ; p=0.89$ for ventral tegmental area], dropping by $22 \%$ relative to the control group.

\section{Glial fibrillary acidic protein immunoreactivity}

GFAP IHC stains both resting and reactive astrocytes in the mouse brain. Resting astrocytes are easily identified in white matter tracts and several nuclei, but not in the striatum. Reactive astrocytes are readily seen in the striatum of combined PQ $+\mathrm{MB}$ animals and rarely seen in saline animals. Reactive astrocytes in the striatum tended to occur in clusters of 2-20. Combined treatment with PQ $+\mathrm{MB}$ resulted in gliosis in the dorsal (Fig. 8) but not the ventral striatum, as indicated by counts of either the total number of reactive astrocytes or total number of clusters of reactive astrocytes (Table 1). The total number of reactive astrocytes was nonsignificantly increased to 169 and $161 \%$ in response to PQ alone and MB alone, respectively, but rose to $373 \%$ in the combined PQ + MB group [main effect of treatment, $F_{(3,19)}=4.63(p=0.0136)$ ] compared with controls. When counted as total numbers of "clusters," the combined PQ + MB group increased the number of clusters relative to those in control, PQ only, and MB only to levels of $275 \%$ of control [main effect of treatment, $F_{(3,20)}=7.14(p=0.0019)$ ].

\section{DISCUSSION}

This study examined the premise that protracted exposure to subclinical or minimally effective doses of PQ and MB could, when combined, produce additive or even synergistic effects by simultaneously impacting multiple target sites of the DA system and precluding homeostatic reregulation. Indeed, combined PQ $+\mathrm{MB}$ produced synergistic effects targeting the nigrostriatal DA system that were progressive in nature and consistent with neurotoxicity, validating the model suggested by our previous findings (Thiruchelvam et al., 2000). Sustained reductions in locomotor activity immediately after injection and failure to evidence full recovery 24 hr later were observed only in the $\mathrm{PQ}+\mathrm{MB}$ group. $\mathrm{PQ}+\mathrm{MB}$, but not either alone, reduced striatal but not nucleus accumbens $\mathrm{TH}$ and DAT immunoreactivity, striatal $\mathrm{TH}$ protein levels, and $\mathrm{TH}$ immunoreactivity and cell counts in the substantia nigra but not in the VTA and produced reactive gliosis in the dorsal striatum but not in the nucleus accumbens. Furthermore, a dose of MPTP (15 $\mathrm{mg} / \mathrm{kg}$ ) without effects in control mice markedly decreased locomotor activity in groups receiving $\mathrm{PQ}, \mathrm{MB}$, or $\mathrm{PQ}+\mathrm{MB}$. Thus, previous exposure to $\mathrm{PQ}$ and/or $\mathrm{MB}$ enhances nigrostriatal system vulnerability to the adverse effects of the selective nigrostriatal neurotoxin MPTP, further confirming adverse effects of PQ and $\mathrm{MB}$ on this system.

These findings also indicate progressive neurotoxicity with continuing exposure. The more protracted exposures used here (12 injections) increased the magnitude of adverse effects relative to those seen after 4 injections (Thiruchelvam et al., 2000). TH 


\section{A TH WESTERN BLOT}

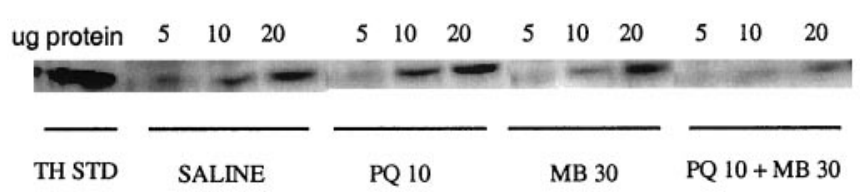

Figure 6. Western blot analysis of TH protein in mouse striatum. A, The autoradiogram depicts striatal samples isolated $5 \mathrm{~d}$ after the last treatment. For each sample, three different concentrations $(5,10$, and $20 \mu \mathrm{g}$ ) of striatal protein were loaded onto separate lanes of the gel. In addition, a known amount of purified TH protein standard (TH STD) was loaded onto the left lane and was used to normalize density units between gels. $B$, Group mean $( \pm \mathrm{SE})$ levels of tyrosine hydroxylase protein levels (plotted as percent of control group values) were measured by Western blot analysis in the dorsal striatum $1 \mathrm{hr}$ or $5 \mathrm{~d}$ after the 12 th injection of vehicle $(C O N T R O L ; n=8), 10 \mathrm{mg} / \mathrm{kg}$ paraquat $(P Q 10 ; n=8), 30 \mathrm{mg} / \mathrm{kg}$ maneb $(M B 30 ; n=8)$, or their combination $(P Q 10+M B 30 ; n=8)$. Bonferroni-Dunn post hoc analysis indicates significant differences: *, from the control group; +, from PQ alone; \#, from $\mathrm{MB}$ alone.

\section{B TYROSINE HYDROXYLASE - WESTERN BLOT ANALYSIS}

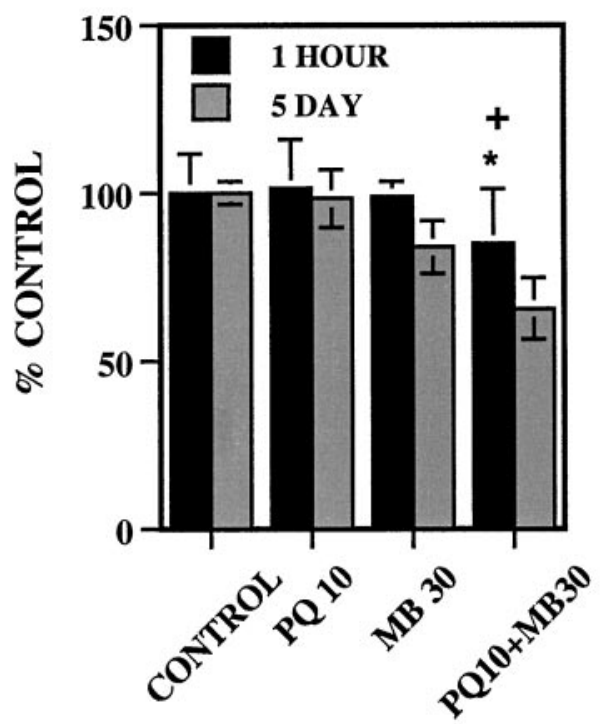

TREATMENT
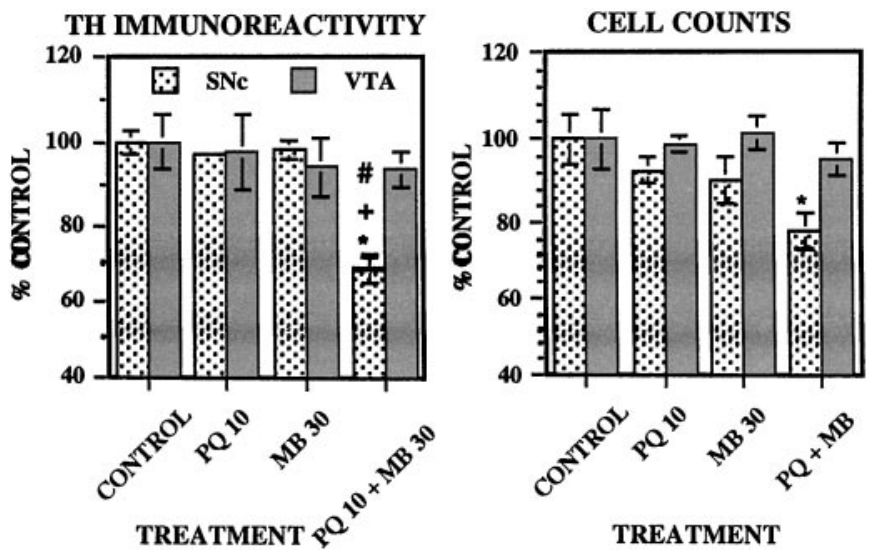

Figure 7. TH immunoreactivity and neuronal cell counts in the substantia nigra and ventral tegmental area. Group mean $( \pm S E)$ levels of TH immunoreactivity (left) and total cell counts (right) were measured $5 \mathrm{~d}$ after the 12 th injection of vehicle $(C O N T R O L ; n=6), 10 \mathrm{mg} / \mathrm{kg}$ paraquat $(P Q 10 ; n=$ $6), 30 \mathrm{mg} / \mathrm{kg}$ maneb $(M B 30 ; n=6)$, or their combination $(P Q 10+M B 30$; $n=6$ ). Bonferroni-Dunn post hoc analysis indicates significant differences: *, from the control group; +, from PQ alone; \#, from MB alone.

density declined $10 \%$ after 4 injections and $17 \%$ after $12 \mathrm{PQ}+\mathrm{MB}$ injections. Although motor activity levels had returned to control values within $24 \mathrm{hr}$ after 4 injections, a failure to recover fully had emerged by treatment 12 . Furthermore, increases in DA and DOPAC tended to remain elevated even $3 \mathrm{~d}$ after 12 injections, whereas corresponding values after 4 injections were below control group values. Moreover, these effects appear to be irreversible; 5 -month-old mice that received six injections of $\mathrm{PQ}+\mathrm{MB}$ showed $39 \%$ reductions in striatal $\mathrm{TH}$ protein levels when measured 3 months after treatment ended. Reversibility, or the extent of recovery, may, however, depend on the age at which exposures are imposed, and ongoing studies will assist in addressing this issue.

The collective findings suggest that $\mathrm{PQ}+\mathrm{MB}$ may be an environmental analog of the methamphetamine model of PD. Repeated methamphetamine produces surges of DA that ultimately reduce DA and metabolites and DA transporter and $\mathrm{TH}$ activity and produces reactive gliosis in the striatum and nigra (Kogan et al., 1976; Sonsalla et al., 1989; O'Dell et al., 1991; Bowyer et al., 1998; Escubedo et al., 1998). In one study, cell loss in the substantia nigra was reported (Sonsalla et al., 1996). Indeed, DA infusions into striatum produce neuronal loss and reactive gliosis (Filloux and Townsend, 1993; Hastings et al., 1996). PQ + MB injections also engendered surges of DA and metabolites and of DA turnover ultimately accompanied by reductions of TH and DAT density and of TH protein in striatum, consistent with terminal dysfunction or more likely a loss of terminals. Either of these outcomes could further elevate DA and metabolite levels. Although the striatum contains serotonergic and cholinergic neurons, the decreases of TH and DAT immunoreactivity in the striatum and of TH immunoreactivity in the substantia nigra argue that GFAP elevations reflect damage to DA components. DA might exert neurotoxic effects via enzymatic metabolism forming $\mathrm{H}_{2} \mathrm{O}_{2}$ that can be broken down to free radical species in the presence of metals (Cohen, 1984; Spina and Cohen, 1989; Olanow and Tatton, 1999) or via auto-oxidation forming various reactive compounds (Halliwell and Gutteridge, 1984). Without direct evidence, however, the possibility that PQ + $\mathrm{MB}$ exposure damages other striatal neurochemical systems cannot yet be excluded.

The surges in DA observed $1 \mathrm{hr}$ to $3 \mathrm{~d}$ after PQ + MB probably underlie the corresponding suppression in motor activity. Indirect DA agonists such as cocaine and amphetamine increase activity at low doses but at higher doses decrease activity, probably reflecting their ability to provoke stereotyped behaviors (Ansah et al., 1993; Rosenzewig-Lipson et al., 1997). Although both PQ and MB alone also increased DA $1 \mathrm{hr}$ after the 12th injection, these effects had a later onset in the course of treatment; they were not evident after four injections as were increases in DA in response to $\mathrm{PQ}+\mathrm{MB}$ (Thiruchelvam et al., 2000) and thus may not have yielded sufficient DA surges to evoke corresponding changes in motor activity or nigrostriatal system neurotoxicity. Interestingly, only $\mathrm{PQ}+\mathrm{MB}$ also resulted in an increase in DA turnover after 12 injections, indicating a more substantial involvement of DA system changes with the combined exposure.

The basis for selective vulnerability of the nigrostriatal system to $\mathrm{PQ}+\mathrm{MB}$ is not yet clear. $\mathrm{PQ}+\mathrm{MB}$ reduced TH and DAT striatal immunoreactivity in dorsal striatum, but not in nucleus accumbens, and reduced $\mathrm{TH}$ immunoreactivity and cell counts in substantia nigra pars compacta, but not in VTA. Reactive gliosis was observed in dorsal striatum but not nucleus accumbens. In PD, pigmented melanized DA neurons are targeted, and there is a greater number of pigmented neurons in substantia nigra $(>80 \%)$ than in VTA (50\%) (Hirsch et al., 1989; Hirsch and Faucheux, 1998). PQ, like MPTP, has a propensity to accumulate in melanincontaining neurons. However little neuromelanin exists in mouse 
SALINE

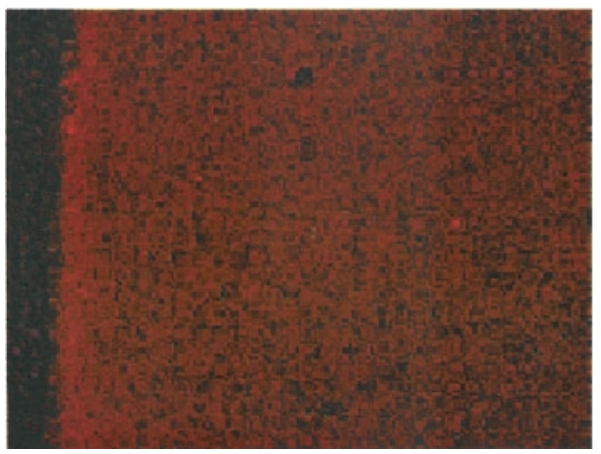

PARAQUAT + MANEB

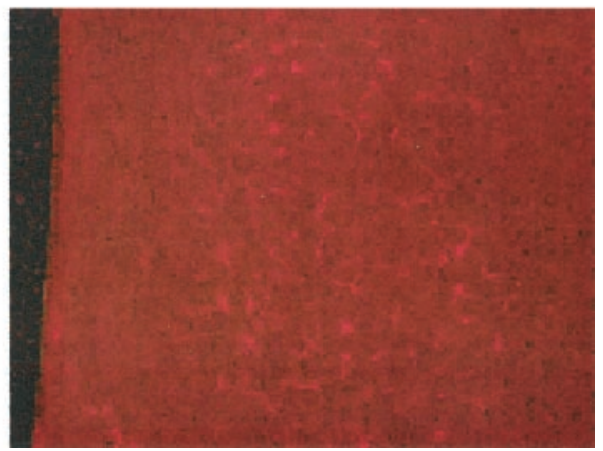

Figure 8. GFAP immunoreactivity in dorsal striatum. Representative photomicrographs depicting GFAP immunoreactivity $5 \mathrm{~d}$ after the 12th injection of vehicle $(A)$ or the combination of $10 \mathrm{mg} / \mathrm{kg}$ paraquat and $30 \mathrm{mg} / \mathrm{kg}$ maneb $(B)$ in dorsal striatum. The combined treatment shows distinguishable clusters of reactive glia that were not observed after corresponding treatment with vehicle or PQ or MB alone.

Table 1. GFAP immunoreactivity in dorsal striatum

\begin{tabular}{lllll} 
Treatment & $\begin{array}{l}\text { Total number of } \\
\text { astrocytes }\end{array}$ & $\begin{array}{l}\text { \% of control number } \\
\text { astrocytes }\end{array}$ & $\begin{array}{l}\text { Total number of } \\
\text { clusters }\end{array}$ & $\begin{array}{l}\% \text { of control number } \\
\text { of clusters }\end{array}$ \\
\hline Saline & $11 \pm 2.2$ & $100 \pm 18.5$ & $3 \pm 0.8$ & $100 \pm 45.1$ \\
Paraquat $(10 \mathrm{mg} / \mathrm{kg})$ & $20 \pm 5.4$ & $169 \pm 46$ & $4 \pm 0.88$ & $130 \pm 26.5$ \\
Maneb $(30 \mathrm{mg} / \mathrm{kg})$ & $19 \pm 4.3$ & $161 \pm 36.5$ & $5 \pm 0.62$ & $140 \pm 18.4$ \\
Paraquat + maneb & $44 \pm 11^{*}$ & $373 \pm 98.2^{*}$ & $9 \pm 1.4^{*} \dagger \#$ & $275 \pm 42^{*} \dagger \#$
\end{tabular}

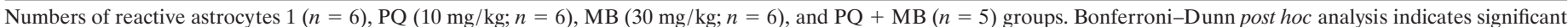
differences from either control paraquat alone or maneb alone.

Significantly different: *, from control; $\uparrow$, from paraquat alone; \#, from maneb alone.

brain (Barden and Levine, 1983), suggesting that other sites must be targeted. The cause(s) for the synergistic effects of PQ + MB cannot be discerned from the current study. One possibility involves alterations in kinetics and consequent brain uptake. Interestingly, diethyldithiocarbamate can increase $\mathrm{MPP}^{+}$levels in brain after MPTP administration (Irwin et al., 1987). It is conceivable that MB, an ethylenebisdithiocarbamate, increases PQ uptake into brain, because $\mathrm{PQ}$ is structurally similar to $\mathrm{MPP}^{+}$. $\mathrm{PQ}$ injected directly into nigra does produce neurotoxicity and nigrostriatal system degeneration (Liou et al., 1996).

PQ + MB could act via several different mechanisms to alter DA function. $\mathrm{PQ}$, by redox cycling, can generate oxygen free radicals (Clejan and Cederbaum, 1989) that exert cytotoxic effects by disrupting mitochondrial complex 1 activity (Fukushima et al., 1993; Tawara et al., 1996). MB can alter vesicular glutamate uptake (Fukushima et al., 1993; Tawara et al., 1996; Vaccari et al., 1998, 1999) and storage and release of striatal DA (Vaccari et al., 1996). The ethylenebisdithiocarbamate anion and not the manganese moiety of MB appears to be the active component (Soleo et al., 1996) and possesses chelating properties. Thus, it could chelate metals such as iron, which have been shown to accumulate in brains of PD patients (Lan and Jiang, 1997). These possibilities are consistent with the assertion that targeting multiple sites of the nigrostriatal DA system may impede homeostatic reregulation, resulting in toxicity.

Doses of PQ and MB used here were not associated with overt toxicity, body weight changes, or lung pathology. The MB dose is $<1 \%$ and the PQ dose is possibly $10-33 \%$ of reported $\mathrm{LD}_{50}$ values (Bus et al., 1976; Berg, 1977; Drew and Gram, 1979; Yamamoto, 1993). Information on actual human exposure levels to these compounds, which may occur via dermal, inhalation, and/or oral exposures, is not readily available. That PQ $+\mathrm{MB}$ exposures could occur concurrently is suggested by overlap in geographical use and the fact that maximal residue levels for both are established for food products. Maneb and mancozeb occur as residues on foods (Newsome, 1976; Patsakos et al., 1992; Yamamoto, 1993). Increases in health complaints related to drifts of paraquat sprays have been reported (Ames et al., 1993). Paraquat is used on cotton, and cotton waste can be used as a food supplement for beef cattle. Although presumably at much lower levels than used here, doses in the current study were imposed over only a 6 week period, unlike the much more protracted exposures that might be expected in human populations. It also seems unlikely that the effects observed here would be restricted to the combination of $\mathrm{PQ}+\mathrm{MB} . \mathrm{MB}$ is only one member of a larger family of dithiocarbamates used in geographical overlap with PQ. Numerous other agrichemicals, including organophosphates, share these patterns of geographical use and are also found as residues in foods. Thus, there is clearly a basis to suppose that supramixtures of agricultural chemical exposures may be likely. Our findings, therefore, may represent only a preliminary assessment of the role of environmental agrichemicals as risk factors for PD.

Evidence from this study also suggests that prolonged exposures to $\mathrm{PQ}$ and $\mathrm{MB}$ alone may result in progressive effects. As exposure progressed, systemic $\mathrm{MB}$ reduced locomotor activity, enhanced DA levels and susceptibility to MPTP, and decreased DA transporter density. Although effects of systemic PQ exposures have been equivocal (Ames et al., 1993; Widdowson et al., 1996a,b), this study appears to be the first to report that a behaviorally silent dose of PQ can potentiate MPTP's effects on motor activity and also ultimately increase DA levels. Effects of PQ alone may be enhanced by blood-brain barrier disruption (Brooks et al., 1999), raising questions about the extent to which such combined exposures either early in development or during advanced age may enhance brain uptake of these agrichemicals. Interestingly, mice exposed to $0.36 \mathrm{mg} / \mathrm{kg}$ PQ at postnatal day 10-11 show permanent hypoactivity and attenuated levels of striatal DA (Fredriksson et al., 1993). Uptake of PQ into brain is age dependent, with higher concentrations of PQ detected in very young (2-weeks-old) and old (24-months-old) animals (Corasaniti et al., 1991; Fredriksson et al., 1993; Widdowson et al., 1996a).

Risk assessment guidelines for human exposure to compounds such as those used here are typically based on levels producing no effect derived from exposures to single agents. Our findings show, however, that such compounds, while having no or marginal effects when administered individually, can produce synergistic effects when given in combination. This obviously implies that the current derivation of risk assessment guidelines needs to be reevaluated. Finally, it is unlikely that such exposures per se produce PD. A more probable etiological basis involves gene-environment interactions, in which such exposures would act in conjunction with a 
susceptible genetic predisposition. Thus future studies imposing such exposures on genetic backgrounds of potential vulnerability could further advance the understanding of PD.

\section{REFERENCES}

Ames RG, Howd RA, Doherty L (1993) Community exposure to a paraquat drift. Arch Environ Health 48:47-52.

Ansah TA, Wade LH, Shockley DC (1993) Effects of calcium channel entry blockers on cocaine and amphetamine-induced motor activities and toxicities. Life Sci 53:1947-1956.

Barden H, Levine S (1983) Histochemical observations on rodent brain melanin. Brain Res Bull 10:847-851.

Berg GL (1977) Farm chemicals handbook. Willoughby, OH: Meister.

Bowyer JF, Frame LT, Clausing P, Nagamoto-Combs K, Osterhout CA, Sterling CR, Tank AW (1998) Long-term effects of amphetamine neurotoxicity on tyrosine hydroxylase mRNA and protein in aged rats. J Pharmacol Exp Ther 286:1074-1085.

Brooks AI, Chadwick C, Gelbard HA, Cory-Slechta DA, Federoff HJ (1999) Paraquat elicited neurobehavioral syndrome caused by dopaminergic neuron loss. Brain Res 27:1-10.

Bus JS, Cagen SZ, Olgaard M, Gibson JE (1976) A mechanism of paraquat toxicity in mice and rats. Toxicol Appl Pharmacol 35:501-513.

Clejan L, Cederbaum AI (1989) Synergistic interactions between NADPH-cytochrome P-450 reductase, paraquat and iron in the generation of active oxygen radicals. Biochem Pharmacol 38:1779-1786.

Cohen G (1984) Oxy-radical toxicity in catecholamine neurons. Neurotoxicology 5:77-82.

Corasaniti MT, Defilippo R, Rodino P, Nappi G, Nistico G (1991) Evidence that paraquat is able to cross the blood brain barrier to a different extent in rats at various ages. Funct Neurol 6:385-391.

Drew R, Gram TE (1979) Vehicle alteration of paraquat lethality in mice. Toxicol Appl Pharmacol 48:479-487.

Escubedo E, Guitart L, Sureda FX, Jimenez A, Pubill D, Pallas M, Camins A, Camarasa J (1998) Microgliosis and down-regulation of adenosine transporter induced by methamphetamine in rats. Brain Res 814:120-126.

Ferraz HB, Bertolucci PHF, Pereira JS, Lima JGC, Andrade LAF (1988) Chronic exposure to the fungicide maneb may produce symptoms and signs of CNS manganese intoxication. Neurology 38:550-553.

Filloux F, Townsend JJ (1993) Pre- and postsynaptic neurotoxic effects of dopamine demonstrated by intrastriatal injection. Exp Neurol 119:79-88.

Fredriksson A, Fredriksson M, Eriksson P (1993) Neonatal exposure to paraquat or MPTP induces permanent changes in striatum dopamine and behavior in adult mice. Toxicol Appl Pharmacol 122:258-264.

Fukushima T, Yamada K, Hojo N, Isobe A, Shiwaku K, Yamane Y (1993) Mechanisms of cytotoxicity of paraquat: NADH oxidation and paraquat radical formation via complex 1. Exp Toxicol Pathol 45:345-349.

Gorell JM, Johnson CC, Rybicki BA, Peterson EL, Richardson RJ (1998) The risk of Parkinson's disease with exposure to pesticides, farming, well water and rural living. Neurology 50:1346-1350.

Halliwell B, Gutteridge MC (1984) Oxygen toxicity, oxygen radicals, transition metals and disease. Biochem J 219:1-14.

Hastings TG, Lewis DA, Zigmond MJ (1996) Role of oxidation in the neurotoxic effects of intrastriatal dopamine injections. Proc Natl Acad Sci USA 93:1956-1961.

Hertzman C, Wiens M, Bowering D, Snow B, Caine D (1990) Parkinson's disease: a case-control study of occupational and environmental risk factors. Am J Ind Med 17:349-355.

Hirsch EC, Faucheux BA (1998) Iron metabolism and Parkinson's disease. Mov Disord 13:39-45

Hirsch EC, Graybiel AM, Agid Y (1989) Selective vulnerability of pigmented dopaminergic neurons in Parkinson's disease. Acta Neurol Scand 126:19-22.

Irwin I, Wu EY, DeLanney LE, Trevor A, Langston JW (1987) The effect of diethyldithiocarbamate on the biodisposition of MPTP: an explanation for enhanced neurotoxicity. Eur J Pharmacol 141:209-217.

Kogan FJ, Nichols WK, Gibb JW (1976) Influence of methamphetamine on nigral and striatal tyrosine hydroxylase activity and on striatal dopamine levels. Eur J Pharmacol 36:363-371.

Lan J, Jiang DH (1997) Excessive iron accumulation in the brain: a possible potential risk of neurodegeneration in Parkinson's disease. J Neural Transm 104:649-660.

Langston JW, Irwin I (1986) MPTP: current concepts and controversies. Clin Neuropharmacol 9:485-507.

Langston JW, Forno LS, Rebert CS, Irwin I (1984) Selective nigral toxicity after systemic administration of MPTP in the squirrel monkey. Brain Res 292:1480-1482.

Liou HH, Chen RC, Tsai YF, Chen WP, Chang YC, Tsai MC (1996) Effects of paraquat on the substantia nigra of the Wistar rats: neurochemical, histological, and behavioral studies. Toxicol Appl Pharmacol 137:34-41.

Liou HH, Tsai MC, Chen CJ, Jeng JS, Chang YC, Chen SY, Chen RC (1997) Environmental risk factors and Parkinson's disease: a casecontrol study in Taiwan. Neurology 48:1583-1588.

Meco G, Bonifati V, Vanacore N, Fabrizio E (1994) Parkinsonism after chronic exposure to the fungicide maneb (manganese ethylene-bisdithiocarbamate. Scand J Work Environ Health 20:301-305.
Miller DB, Reinhard Jr JF, Daniels AJ, O'Callaghan JP (1991) Diethyldithiocarbamate potentiates the neurotoxicity of in vivo 1-methyl-4phenyl-1,2,3,6-tetrahydropyridine and of in vitro 1-methyl-4-phenylpyridinium. J Neurochem 57:541-549.

Morato GS, Lemos T, Takahashi RN (1989) Acute exposure to maneb alters some behavioral functions in the mouse. Neurotoxicol Teratol $11: 421-425$

Newsome WH (1976) Residues of four ethylenebis (dithiocarbamates) and their decomposition products on field-sprayed tomatoes. J Agric Food Chem 24:999-1001.

O’Dell SJ, Weihmuller FB, Marshall JF (1991) Multiple methamphetamine injections induce marked increases in extracellular striatal dopamine which correlate with subsequent neurotoxicity. Brain Res 564:256-260.

Olanow CW, Tatton WG (1999) Etiology and pathogenesis of Parkinson's disease. Annu Rev Neurosci 22:123-144.

Osterhout CA, Chikaraishi DM, Tank AW (1997) Induction of tyrosine hydroxylase protein and a transgene containing tyrosine hydroxylase $5^{\prime}$ flanking sequences by stress in mouse adrenal gland. J Neurochem 68:1071-1077.

Patsakos PG, Liapis K, Miliadis GE, Zafiriou K (1992) Mancozeb residues of field sprayed apricots. Bull Environ Contam Toxicol 48:756-761.

Rajput AH, Uitti RJ, Stern W, Laverty W, O'Donnell K, O'Donnell D, Yuen WK, Dua A (1987) Geography, drinking water chemistry, pesticides and herbicides and the etiology of Parkinson's disease. Can J Neurol Sci 14:414-418.

Rosenzewig-Lipson S, Thomas S, Barrett JE (1997) Attenuation of the locomotor activating effects of D-amphetamine, cocaine and scopolamine by potassium channel modulators. Prog Neuropsychopharmacol Biol Psychiatry 21:853-872.

Semchuk KM, Love EJ, Lee RG (1992) Parkinson's disease and exposure to agricultural work and pesticide chemicals. Neurology 42:1328-1335.

Soleo L, Defazio G, Scarselli R, Zefferino R, Livrea P, Foa V (1996) Toxicity of fungicides containing ethylene-bis-dithiocarbamate in serumless dissociated mesencephalic-striatal primary co-culture. Arch Toxicol 70:678-682.

Sonsalla PK, Nicklas WJ, Heikkila RE (1989) Role for excitatory amino acids in methamphetamine-induced nigrostriatal dopaminergic toxicity. Science 243:398-400.

Sonsalla PK, Jochnowitz ND, Zeevalk GD, Oostveen JA, Hall ED (1996) Treatment of mice with methamphetamine produces cell loss in the substantia nigra. Brain Res 738:172-175.

Spina MB, Cohen G (1989) Dopamine turnover and glutathione oxidation: implications for Parkinson's disease. Proc Natl Acad Sci USA 86:1398-1400.

Takahashi RN, Rogerio R, Zanin M (1989) Maneb enhances MPTP neurotoxicity in mice. Res Commun Chem Pathol Pharmacol 66:167-170.

Tanner CM, Ben-Shlomo Y (1999) Epidemiology of Parkinson's disease. In: Parkinson's disease: advances in neurology (Stern GM, ed), pp 153-159. Philadelphia: Lippincott, Williams, and Wilkins.

Tanner CM, Ottman R, Goldman SM, Ellenberg J, Chan P, Mayeux R, Langston JW (1999) Parkinson disease in twins: an etiologic study. JAM A 281:341-346.

Tawara T, Fukushima T, Hojo N, Isobe A, Shiwaku K, Setogawa T, Yamane Y (1996) Effects of paraquat on mitochondrial electron transport system and catecholamine contents in rat brain. Arch Toxicol 70:585-589.

Thiruchelvam M, Brockel BJ, Richfield EK, Baggs RB, Cory-Slechta DA (2000) Potentiated and preferential effects of combined paraquat, maneb on nigrostriatal dopamine systems: environmental risk factors for Parkinson's disease? Brain Res 873:225-234.

United States Geographic Service (1998) Pesticide National Synthesis Project. Washington, DC: USGS.

Vaccari A, Saba PL, Ruiu S, Collu M, Devoto P (1996) Disulfiram and diethyldithiocarbamate intoxication affects the storage and release of striatal dopamine. Toxicol Appl Pharmacol 139:102-108.

Vaccari A, Ferraro L, Saba P, Ruiu S, Mocci I, Antonelli T, Tanganelli S (1998) Differential mechanisms in the effects of disulfiram and diethyldithiocarbamate intoxication on striatal release and vesicular transport of glutamate. J Pharmacol Exp Ther 285:961-967.

Vaccari A, Saba P, Mocci I, Ruiu S (1999) Dithiocarbamate pesticides affect glutamate transport in brain synaptic vesicles. J Pharmacol Exp Ther 288:1-5.

Walters TL, Irwin I, Delfani K, Langston JW, Janson AM (1999) Diethyldithiocarbamate causes nigral cell loss and dopamine depletion with nontoxic doses of MPTP. Exp Neurol 156:62-70.

Widdowson PS, Farnworth MJ, Simpson MG, Lock EA (1996a) Influence of age on the passage of paraquat through the blood-brain barrier in rats: a distribution and pathological examination. Hum Exp Toxicol 15:231-236.

Widdowson PS, Farnworth MJ, Upton R, Simpson MG (1996b) No changes in behaviour, nigro-striatal system neurochemistry or neuronal cell death following toxic multiple oral paraquat administration to rats. Hum Exp Toxicol 15:583-591.

Wilhoit L, Davidson N, Supkoff D, Stegall J, Braun A, Simmons S, Hobza B, Hunter C, Gorder N, Goodman C, Todd B (1999) Pesticide use analysis and trends from 1991 to 1996. Sacramento, CA: State of California Environmental Protection Agency.

Yamamoto HA (1993) Protection against paraquat-induced toxicity with sulfite or thiosulfate in mice. Toxicology 79:37-43. 